\title{
UTILIZAÇÃO DE MACRÓFITAS MARINHAS NO MONITORAMENTO DA CONTAMINAÇÃO POR METAIS PESADOS: O CASO DA BAÍA DE SEPETIBA, RJ
}

\author{
Gilberto M. Amado Filho ${ }^{1}$ \\ Wolfgang C. Pfeiffer ${ }^{2}$
}

Recebido em 12/01/1999. Aceito em 17/05/1999

\begin{abstract}
RESUMO - (Utilização de macrófitas marinhas no monitoramento da contaminação por metais pesados: o caso da Baía de Sepetiba, RJ). A viabilidade de utilização de macrófitas marinhas para o monitoramento da contaminação por metais pesados em ambientes costeiros foi avaliada através de análises das concentrações de alguns metais em duas espécies de algas pardas, Padina gymnospora e Sargassum stenophyllum, e numa espécie de monocotiledônea marinha, Halodule wrightii. Essas espécies foram coletadas em duas regiōes do Estado do Rio de Janeiro, a Baía de Sepetiba, que vem sofrendo processo de degradação ambiental crescente nas últimas décadas, e a Baía da Ribeira, não contaminada. Foram também realizados experimentos de transplante in situ com $P$. gymnospora entre essas duas regiões. As duas espécies de algas analisadas apresentam comportamento semelhante quanto à acumulação de metais. As concentrações nas algas indicaram distribuição espacial ampla dos metais $\mathrm{Zn}$ e Cd na Baía de Sepetiba, sempre mais elevadas do que na Baía da Ribeira, e evolução temporal não sazonal e sim relacionada com a carga de lançamentos de metais para a Baía. Os resultados do experimento de transplante mostraram que $P$. gymnospora integra relativamente rápido as concentrações biodisponíveis de metais no meio, quando transplantadas de local não contaminado para outro contaminado, e que a eliminação de metais da planta para o meio é pouco expressiva no local não contaminado. Em relação à monocotiledônea $H$. wrightii, evidenciou-se que as raízes e folhas são os principais compartimentos para acumulação de metais e que concentrações elevadas nas raízes encontradas nas populações da Baía de Sepetiba podem refletir a biodisponibilidade de metais no sedimento.
\end{abstract}

Palavras-chave - metais pesados, ecossistemas costeiros, Padina gymnospora, Sargassum stenophyllum, Halodule wrightii

\begin{abstract}
The use of marine macrophytes to monitor heavy metal contamination: the case of Sepetiba Bay, RJ). The viability of marine macrophytes being used as monitors of heavy metal contamination in coastal zones was evaluated by analysis of the concentration of certain elements in two brown algae species, Padina gymnospora and Sargassum stenophyllum, and in a seagrass species, Halodule wrightii. These species were collected in two Rio de Janeiro State regions, Sepetiba Bay, which has been the brunt of an environmental degradation process over the last decades, and Ribeira Bay, a noncontaminated area. In addition, in situ transplant experiments with $P$. gymnospora between the two regions were done. The two algal species presented a similar behavior regarding heavy metal accumulation,
\end{abstract}

1 Programa Zona Costeira, Instituto de Pesquisas Jardim Botânico do Rio de Janeiro, Rua Pacheco Leão 915, CEP 22460-030, Rio de Janeiro, RJ, Brasil. e-mail: gfilho@jbrj.gov.br

2 Laboratório de Radioisótopos, Instituto de Biofísica Carlos Chagas Filho, CCS, Universidade Federal do Rio de Janeiro, CEP 22490-090, Rio de Janeior, RJ, Brasil 
indicating high concentrations of $\mathrm{Zn}$ and $\mathrm{Cd}$ in Sepetiba Bay in relation to Ribeira Bay, a wide distribution of these metals along Sepetiba Bay, and a non seasonal temporal variation of heavy metal levels, this being related to the inputs of the metal throughout into the Bay. The results of transplant experiments show that $P$. gymnospora is able to accumulate the studied metals in a short period of time (one month) thus reflecting the ambient bioavailability in Sepetiba Bay, and a low plant metal release in the noncontaminated region. In relation to the seagrass $H$. wrightii roots and leaves were the main compartments to metal accumulation and the high metal concentration in the roots found in Sepetiba Bay populations could reflect the metal bioavailability in sediments.

Key words - heavy metals, coastal ecosystems, Padina gymnospora, Sargassum stenophyllum, Halodule wrightii

\section{Introdução}

$\mathrm{O}$ aumento crescente dos problemas relacionados à poluição, causados principalmente por despejos industriais, tem levado a ações no sentido de se estimar os efeitos dos poluentes sobre as comunidades marinhas. Dentre os poluentes lançados no meio marinho, destacam-se os metais pesados e seus compostos, introduzidos no meio através de diferentes fontes de origem antropogênica, como rejeitos industriais, domésticos e também por emissões atmosféricas.

Em especial, uma região do Estado do Rio de Janeiro, a Baía de Sepetiba, situada a $60 \mathrm{~km}$ a oeste da cidade do Rio de Janeiro, tem sido caracterizada como contaminada por metais pesados. A principal fonte poluidora desta baía é uma indústria beneficiadora de zinco. Acrescido a este problema já existente, outros fatores determinam a aceleração do processo de degradação ambiental da Baía de Sepetiba, principalmente os relacionados à dragagem do sedimento de fundo para a ampliação do Porto de Sepetiba (Multiservice 1997). A dragagem resultou em significativa remobilização dos metais depositados no sedimento para áreas da baía ainda não atingidas diretamente pelos efluentes industriais, como a região do entorno da Ilha de Jaguanúm (Fig. 1).

Os níveis de metais em algumas espécies de macroalgas marinhas, especialmente algumas feofíceas, podem atingir concentrações até 20 vezes superiores quando comparados com regiões consideradas não contaminadas, sendo indicadas para o monitoramento de metais pesados na Baía de Sepetiba e outras regiões do litoral brasileiro (Amado Filho et al. 1997a; 1997b; 1997c; Karez et al. 1994a; 1994b).

Outro grupo vegetal que ocorre em ambientes marinhos e estuarinos, as monocotiledôneas possuem duas vias distintas para acumulação de elementos essenciais (ou não), as raízes e as folhas, sendo que as raízes absorvem os elementos disponíveis na água intersticial do sedimento (Lacerda \& Rezende 1986). Assim como as macroalgas, essas plantas podem refletir as concentrações de metais no ambiente (Pulich 1980; 1982; Lacerda \& Rezende 1986).

O objetivo deste trabalho é o de demonstrar a viabilidade da utilização de macrófitas marinhas no monitoramento de metais pesados em ecossistemas costeiros estuarinos e marinhos, através da análise da concentração de metais em duas espécies de algas pardas (Phaeophyceae), Padina gymnospora (Kützing) Sonder (Dictyotaceae) e Sargassum stenophyllum Mart. (Sargassaceae) e uma espécie de monocotiledônea marinha, Halodule wrightii Aschers. (Cymodoceaceae), em locais contaminados e não contaminados, bem como por intermédio de experimentos de transplante in situ. 

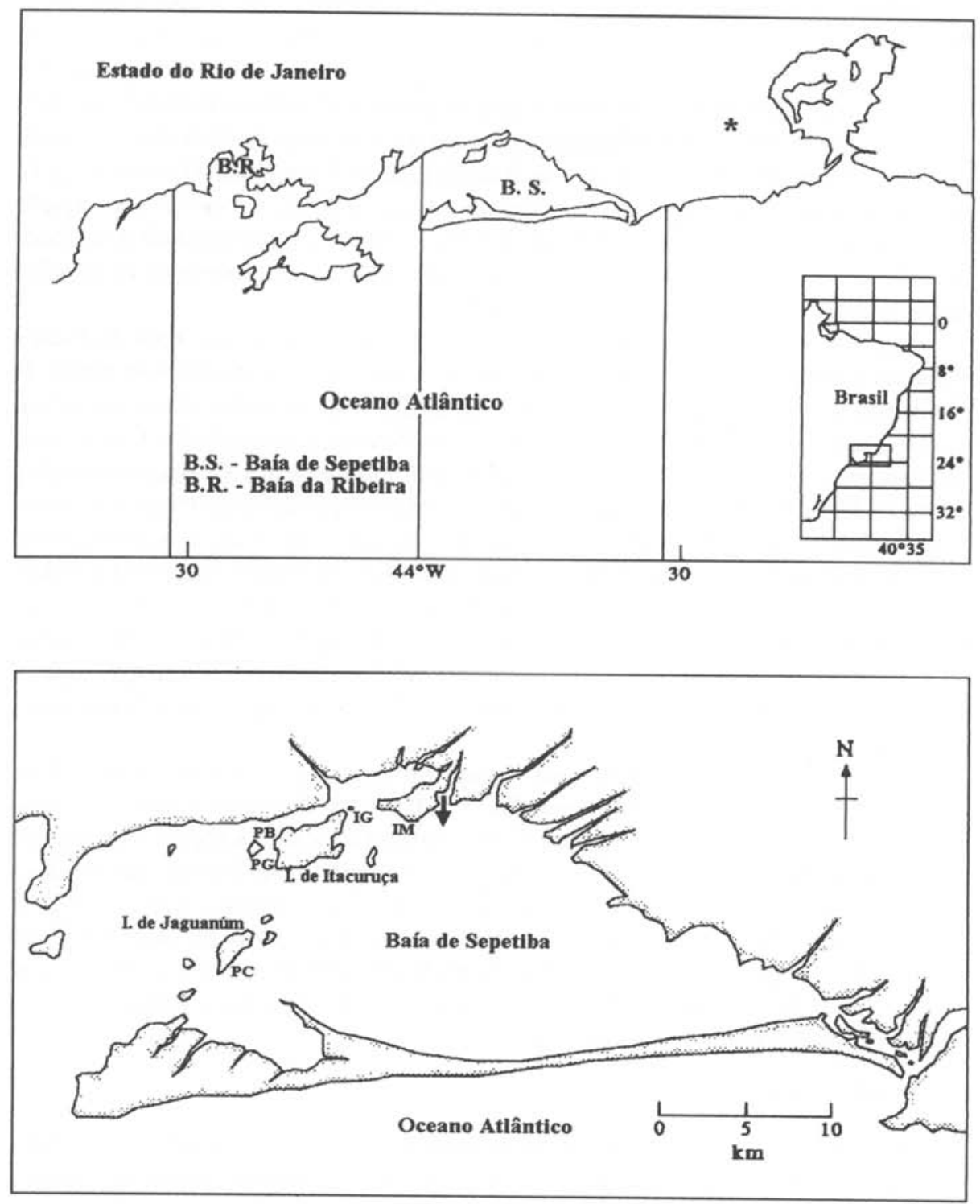

Figura 1. Mapa com a localização das Baías de Sepetiba e Ribeira na costa do Estado do Rio de Janeiro. (*) Cidade do Rio de Janeiro. Localização dos pontos de coleta na Baía de Sepetiba: IM, Ilha da Madeira; IG, Ilha do Gato; PB, Ponto do Boizinho; PG, Praia Grande; PC, Praia do Catita. Seta indica o local da principal fonte de contaminação da Baía de Sepetiba. 


\section{Material e métodos}

Monitoramento de metais pesados na Baía de Sepetiba - as amostras das algas Padina gymnospora e Sargassum stenophyllum foram coletadas em cinco locais da Baía de Sepetiba (Ilha da Madeira, Ilha do Gato, Ponta do Boizinho, Praia Grande e Praia do Catita) e um local da Baía da Ribeira (Piquarara de Fora), enquanto as amostras de Halodule wrightii foram coletadas em dois locais da Baía de Sepetiba (Ponta do Boizinho e Praia do Catita) e um local da Baía da Ribeira (Piraquara de Dentro) (Fig.1). As coletas de algas foram realizadas no período de agosto/1996 a julho/1997. A variação temporal das concentrações de metais desde 1990 à 1997 foi avaliada através de análises em indivíduos de $P$. gymnospora coletados na Ilha do Gato. As coletas de $H$. wrightii foram realizadas em abril, junho e setembro/1997.

Após as coletas, o material foi triado, lavado várias vezes com água do local e posteriormente lavado rapidamente em água destilada e seco a temperatura média de $70^{\circ} \mathrm{C}$ até peso constante. Para $H$. wrightii foram separadas as raízes, rizomas e folhas. Frações de $0,5 \mathrm{~g}$ (peso seco) foram digeridas em ácido nítrico concentrado. Esta solução foi evaporada e o resíduo dissolvido em $10 \mathrm{ml}$ de ácido clorídrico $(0,1 \mathrm{~N})$. As concentrações de metais foram medidas por espectrofotometria de absorção atômica de chama (Varian AA-1475) em triplicata, e os resultados expressos em $\mu \mathrm{g} \cdot \mathrm{g}^{-1}$ (peso seco). A eficiência do procedimento analítico foi determinada através de análise comparativa da amostra padrão de referência da Agência Internacional de Energia Atômica, IAEA - 140 ("sea plant homogenate", Fucus). Foram analisados $\mathrm{Cd}, \mathrm{Cr} \mathrm{e} \mathrm{Zn}$ nas algas e $\mathrm{Cd}$ e $\mathrm{Zn}$ em H. wrightii.

Para comparação dos resultados e determinação das possíveis diferenças entre os locais de coleta utilizou-se a análise de variância (ANOVA) do tipo "one way analysis".

Experimento de transplante com Padina gymnospora - cerca de 25 indivíduos adultos de P. gymnospora foram coletados no infralitoral da Praia Grande, fixos pela base em uma tela de nylon e imediatamente transportados para a região controle não contaminada, Baía da Ribeira. O conjunto, tela de nylon mais plantas, foi fixado a blocos de pedra no infralitoral. Por outro lado, pelo mesmo método, indivíduos de $P$. gymnospora da Baía da Ribeira foram transferidos para a Baía de Sepetiba (Praia Grande). Esse experimento foi realizado em três épocas do ano, setembro/1996, março/ 1997 e junho/1997. Após 30 dias, os indivíduos foram retirados e preparados para análise de metais, conforme descrito acima.

\section{Resultados e discussão}

Concentração de metais nas duas espécies de algas - os resultados verificados mostram que as duas espécies analisadas apresentam comportamento semelhante quanto a acumulação de metais. Os resultados de Cd e Zn em Padina (Tab.1) e em Sargassum (Tab. 2) foram sempre significativamente $(\mathrm{p}<0,05)$ mais elevados em Sepetiba do que no ponto controle (Baía da Ribeira). Os valores encontrados para o $\mathrm{Cr}$ não foram significativamente distintos aos da Ribeira e encontram-se numa faixa característica de regiões tropicais e subtropicais não contaminadas (Amado Filho et al. 1997b; Lacerda et al. 1985). Este elemento apresenta comportamento diferenciado em relação ao $\mathrm{Zn}$ e 
Tabela 1. Concentração média de metais (Cd, $\mathrm{Cr}$ e Zn em $\mu \mathrm{g} \cdot \mathrm{g}^{-1}$ de peso seco) no período de agosto/1996 a julho/1997 em Padina gymnospora de cinco locais da Baía de Sepetiba (IM = Ilha da Madeira, IG = Illa do Gato, PB = Ponta do Boizinho, $\mathrm{PG}=$ Praia Grande, $\mathrm{PC}=$ Praia do Catita $)$ e do local controle $(\mathrm{BR}=$ Baia da Ribeira).

\begin{tabular}{|c|c|c|c|c|c|c|}
\hline \multirow[t]{2}{*}{ Metal } & \multicolumn{6}{|c|}{ Locais de coleta } \\
\hline & IM & IG & PB & PG & PC & BR \\
\hline$\overline{\mathrm{Cd}}$ & $1,8(0,38)$ & $1,2(0,34)$ & $0,96(0,30)$ & $0,94(0,20)$ & $0,75(0,12)$ & $0,30(0,09)$ \\
\hline $\mathrm{Cr}$ & $3,4(0,6)$ & $3,2(0,4)$ & $4,3 \quad(0,7)$ & $4,8 \quad(0,5)$ & $3,3 \quad(0,4)$ & $3,0 \quad(0,6)$ \\
\hline $\mathrm{Zn}$ & $360,0(40)$ & $350,0(38)$ & $248,0 \quad(28)$ & $245,0 \quad(25)$ & $210,0 \quad(23)$ & $23,0 \quad(8)$ \\
\hline
\end{tabular}

Valores entre parênteses $=$ desvio padrão.

Tabela 2. Concentração média de metais ( $\mathrm{Cd}, \mathrm{Cr}$ e $\mathrm{Zn}$ em $\mu \mathrm{g} \cdot \mathrm{g}^{-1}$ de peso seco) no período de agosto de 96 a julho de 97 em Sargassum stenophyllum de cinco locais da Baia de Sepetiba (IM = Ilha da Madeira, IG = Ilha do Gato, $\mathrm{PB}=$ Ponta do Boizinho, $\mathrm{PG}=$ Praia Grande, $\mathrm{PC}=$ Praia do Catita $)$ e no local controle $(\mathrm{BR}=$ Baia da Ribeira).

\begin{tabular}{|c|c|c|c|c|c|c|c|}
\hline \multirow[t]{2}{*}{ Metal } & \multicolumn{7}{|c|}{ Locais de coleta } \\
\hline & $\mathrm{IM}$ & IG & PB & $\mathrm{P}$ & & $\mathrm{PC}$ & BR \\
\hline$\overline{\mathrm{Cd}}$ & * & $1,1 \quad(0,22)$ & $0,78(0,12)$ & 0,62 & $(0,14)$ & $0,48(0,08)$ & $0,20(0,06)$ \\
\hline $\mathrm{Cr}$ & * & $3,1 \quad(0,2)$ & $5,2 \quad(0,8)$ & 4,8 & $(0,7)$ & $3,4 \quad(0,6)$ & $2,7 \quad(0,6)$ \\
\hline $\mathrm{Zn}$ & * & 320,0 (41) & $156,0 \quad(22)$ & 144,0 & (18) & $164,0 \quad(28)$ & $17,0 \quad(4)$ \\
\hline
\end{tabular}

Valores entre parênteses $=$ desvio padrão. ${ }^{*}$ Espécie não encontrada neste local.

Cd na Baía de Sepetiba; enquanto os dois últimos elementos estão em grande parte fracamente ligados ao material particulado em suspensão, ou seja, potencialmente biodisponíveis, o percentual de $\mathrm{Cr}$ na fração fracamente ligada é bem menor e, conseqüentemente, pouco disponível para a biota (Lacerda et al. 1987).

As concentrações de Cd em Padina estão em média 2,5 a 6,0 vezes mais elevadas na Baía de Sepetiba, dependendendo do local de coleta, do que o encontrado no ponto controle, enquanto que em Sargassum, os resultados foram em média de 2,4 a 5,5 vezes mais elevados. Para o $\mathrm{Zn}$, os resultados foram em média de 9,1 a 15,6 vezes mais elevados em Padina e 9,5 a 18,8 vezes mais elevados em Sargassum, respectivamente, em Sepetiba do que na Baía da Ribeira.

As concentrações de $\mathrm{Zn}$ e $\mathrm{Cd}$ nas duas espécies de algas analisadas mantiveram-se elevadas na Baía de Sepetiba, mesmo em locais distantes cerca de $15 \mathrm{~km}$ (Praia do Catita, Ilha de Jaguanúm, Tab. 1 e 2) da principal fonte de lançamento de metais (Fig.1), o que pode estar relacionado à formação de cloro-complexos na água que potencializam o transporte desses elementos para regiões adjacentes (Barcellos et al. 1991). Não foi encontrado padrão sazonal ou tendência a elevação temporal desde 1990 até 1997. Isto pode ser exemplicado pelos resultados de concentrações de metais em Padina na Ilha do Gato (Tab. 3) desde o ano de 1990 até 1997. Entretanto, deve-se destacar que, em determinadas datas, as concentrações foram muito mais elevadas ( $\mathrm{Zn}$ em fevereiro/1996) ou muito mais reduzidas (Zn em agosto/1996) (Tab. 3) do que a concentração média geral .

Esta variação deve-se relacionar, não a um fator ambiental sazonal (salinidade, matéria orgânica em suspensão, estado fisiológico da alga, etc.) que poderia determinar a maior ou menor biodisponibilidade do metal (Riget et al. 1995), mas sim, à maior ou menor carga de lançamentos dos rejeitos da indústria beneficiadora de $\mathrm{Zn}$ no corpo d'agua da Baía de Sepetiba. Isto pode ser comprovado pela relação entre aumento das 
concentrações de $\mathrm{Zn}$ em fevereiro/1996 $\left(899+143 \mu \mathrm{g} \cdot \mathrm{g}^{-1}\right.$, Tab. 3) com o transbordamento para a Baía dos rejeitos da industria ocorrido 20 dias antes, e pela correlação positiva (coeficente de correlação de Spearman, r = 0,62, p < 0,05, n = 12) entre as concentrações de Zn e Cd no período 1990 - 1997 (Tab. 3).

Tabela 3. Concentrações (média e desvio padrão) de Zn e Cd em Padina gymnospora na Illha do Gato (Baía de Sepetiba) entre abril/1990 e julho/1997.

\begin{tabular}{lcc}
\hline Mês/ ano da coleta & \multicolumn{3}{c}{ Metal $\left(\mu \mathrm{g} \cdot \mathrm{g}^{-1}\right.$ de peso seco) } \\
\hline $04 / 1990$ & $470(79)$ & $\mathrm{Cd}$ \\
$05 / 1991$ & $465(41)$ & $1,33(0,22)$ \\
$11 / 1993$ & $541(219)$ & $2,70(0,42)$ \\
$08 / 1995$ & $580(105)$ & $1,63(0,53)$ \\
$02 / 1996$ & $899(134)$ & $1,6(0,50)$ \\
$08 / 1996$ & $166(16)$ & $1,82(0,70)$ \\
$09 / 1996$ & $310(45)$ & $1,22(0,10)$ \\
$10 / 1996$ & $290(40)$ & $1,51(0,12)$ \\
$03 / 1997$ & $320(20)$ & $1,10(0,08)$ \\
$04 / 1997$ & $269(47)$ & $1,05(0,16)$ \\
$06 / 1997$ & $294(34)$ & $1,23(0,09)$ \\
$07 / 1997$ & $380(50)$ & $0,98(0,05)$ \\
& & $1,06(0,08)$ \\
\hline
\end{tabular}

Transplantes com Padina gymnospora - os resultados das concentrações de metais são apresentados na Tab. 4. Após um mês, o $\mathrm{Zn}$ acumulado pelas plantas transplantadas da Baía da Ribeira para a Baía de Sepetiba variaram de 70 a $98 \%$ do encontrado nas populações naturais de Sepetiba (resultados não apresentados neste trabalho), as variações nos níveis de acumulação das plantas transplantadas $\left(70 \pm 14 \mu \mathrm{g} \cdot \mathrm{g}^{1}\right.$, março/ 1997 até $283 \pm 34 \mu \mathrm{g} . \mathrm{g}^{-1}$, junho/1997, Tab. 4) foram dependentes dos níveis encontrados nas populações naturais. Em relação ao $\mathrm{Cd}$, as plantas tranplantadas acumularam no período de 46 a $77 \%$ das concentrações encontradas nas populações de Sepetiba. Diferentemente do $\mathrm{Zn}$, as variações encontradas nos níveis de $\mathrm{Cd}$ entre as épocas do transplante $\left(0,37 \pm 0,10 \mu \mathrm{g} \cdot \mathrm{g}^{-1}\right.$, setembro/1996 a 0,70 $\pm 0,10 \mu \mathrm{g} \cdot \mathrm{g}^{-1}$, março/1997, Tab. 4) foram independentes das encontradas nas populações naturais. As plantas transplantadas para a Baía da Ribeira (Tab. 4) apresentaram, respectivamente, redução de 17 a $23 \%$ nas concentrações de $\mathrm{Zn}$ e de 14 a $37 \%$ de $\mathrm{Cd}$ em relação às populações da Baía de Sepetiba, de onde foram retiradas.

Tabela 4. Concentração de zinco e cádmio em Padina gymnospora após um mês de transplante da Baía de Sepetiba (Praia Grande) para a Baía da Ribeira e vice-versa, em três épocas do ano. Resultados expressos em $\mu \mathrm{g} . \mathrm{g}^{-1}$ de peso seco.

Metal

Períodos dos

$\mathrm{Cd} \quad \mathrm{Zn}$

Transplantes Direção do transplante

\begin{tabular}{lccrc}
\hline (Mes/Ano) & $\begin{array}{c}\text { Ribeira para } \\
\text { Sepetiba }\end{array}$ & $\begin{array}{c}\text { Sepetiba para } \\
\text { Ribeira }\end{array}$ & $\begin{array}{c}\text { Ribeira para } \\
\text { Sepetiba }\end{array}$ & $\begin{array}{c}\text { Sepetiba para } \\
\text { Ribeira }\end{array}$ \\
\hline $09 / 1996$ a $10 / 1996$ & $0,37(0,10)$ & $0,61(0,22)$ & $153(23)$ & $194(25)$ \\
$03 / 1997$ a 04/1997 & $0,70(0,01)$ & $0,81(0,10)$ & $70(14)$ & $142(11)$ \\
$06 / 1997$ a $07 / 1997$ & $0,68(0,02)$ & $0,54(0,04)$ & $283(34)$ & $230(24)$ \\
\hline
\end{tabular}


Verifica-se através deste experimento que $P$. gymnospora integra relativamente rápido as concentrações biodisponíveis de metais no meio, quando transplantadas de um local não contaminado para outro contaminado e que a eliminação do metal da planta para o meio é pouco expressiva, considerando-se que no local não contaminado houve redução na proporção massa de metais/massa nas plantas transplantadas, devido ao crescimento da alga em meio não contaminado. Eide et al. (1980), realizando experimento de transplante semelhante com a alga parda Ascophyllum nodusum, de ambiente temperado, concluíram que o padrão de acumulação de metais pelas plantas está relacionado diretamente à diminuição ou aumento das concentrações de metais na água.

Concentração de metais em Halodule wrigthii - as concentrações de Cd foram sempre mais elevadas nas raízes do que nos outros compartimentos da planta (rizoma e folha) em todas as amostras analisadas (Tab. 5). Quando comparadas as concentrações nas raízes nos diferentes locais, verifica-se que as concentrações nos dois locais da Baía de Sepetiba são significativamente $(\mathrm{p}<0,05)$ mais elevadas do que as encontradas na Baía da Ribeira, e que as concentrações na Praia do Catita foram em média de 2,0 a 2,9 mais elevadas do que as encontradas na Ponta do Boizinho (Tab. 5). Esses resultados sugerem efeito do "bota-fora" do sedimento dragado nas concentrações elevadas de $\mathrm{Cd}$ observadas na Praia do Catita. A maior acumulação de $\mathrm{Cd}$ pelas raízes pode ser explicada pela capacidade que estas plantas apresentam de translocação de oxigênio das folhas para as raízes, resultando deste processo a liberação de oxigênio para o sedimento adjacente, oxidando os compostos reduzidos e precipitando-os na rizosfera (Thursby 1984; Lacerda \& Rezende 1986).

Tabela 5. Concentração de Cd em Halodule wrightii (raiz, rizoma e folha) coletadas em abril, junho e setembro/1997 na Baía de Sepetiba (Praia do Catita e Ponta do Boizinho) e na Baía da Ribeira.

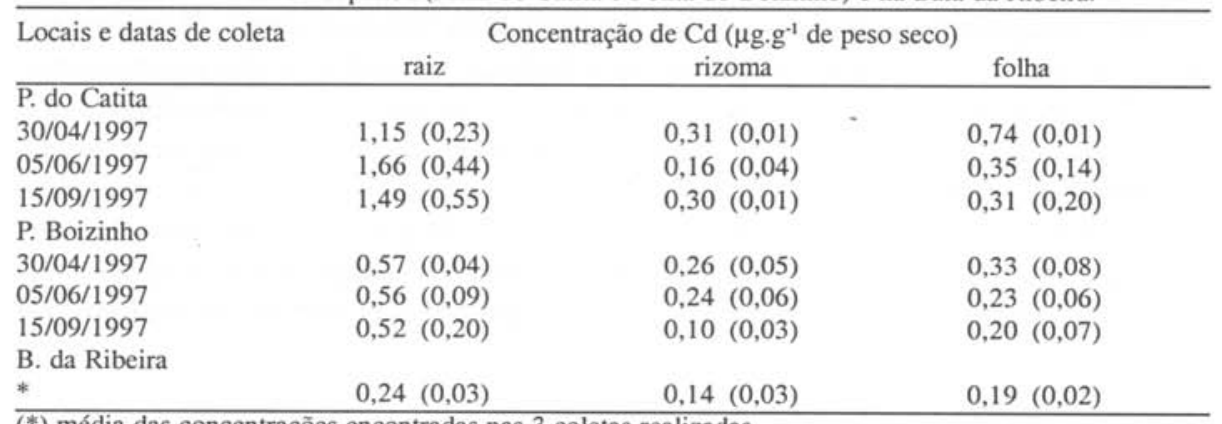

(*) média das concentrações encontradas nas 3 coletas realizadas.

Em relação ao $\mathrm{Zn}$, pode-se observar que as folhas e raízes foram os compartimentos da planta que apresentaram as concentrações mais elevadas em ambos os locais da Baía de Sepetiba, com tendência à maior acumulação pelas folhas (Tab. 6). As concentrações nas raízes (2,1 a 3,9 vezes) e folhas (2,7 a 3,4 vezes) de plantas provenientes dos locais da Baía de Sepetiba foram sempre significativamente ( $\mathrm{p}<$ 0,05) mais elevadas do que as encontradas na Baía da Ribeira (Tab. 6). Em oposição ao 
$C d$, não foram encontradas diferenças significativas $(p>0,05)$ entre as concentrações de $\mathrm{Zn}$ nas raízes e folhas da Praia do Catita e os mesmos compartimentos das plantas provenientes da Ponta do Boizinho. Tem sido indicado que as concentrações de $\mathrm{Zn}$ em monocotiledôneas marinhas são mais elevadas nas folhas (Pulich 1980; Nicolaidou \& Nott 1998) e que este metal é absorvido diretamente da coluna d'agua, entretanto, concentrações elevadas nas raízes, como a observada na Ponta do Boizinho em junho, usualmente refletem circunstâncias de contaminação do sedimento.

Tabela 6. Concentração de $\mathrm{Zn}$ em Halodule wrightii (raiz, rizoma e folha) coletadas em abril, junho e setembro/1997 na Baía de Sepetiba (Praia do Catita e Ponta do Boizinho) e na Baía da Ribeira.

\begin{tabular}{lrrrr}
\hline Locais e datas de coleta & \multicolumn{3}{c}{$\begin{array}{c}\text { Concentração de } \mathrm{Zn}\left(\mu \mathrm{g} . \mathrm{g}^{-1} \text { de peso seco) }\right. \\
\text { rizoma }\end{array}$} \\
\hline $\begin{array}{l}\text { P. do Catita } \\
30 / 04 / 1997\end{array}$ & $77(6,4)$ & $28(1,4)$ & $108(2,1)$ \\
$05 / 06 / 1997$ & $79(7,7)$ & $38(1,4)$ & $98(1,0)$ \\
$15 / 09 / 1997$ & $111(1,4)$ & $43(3,1)$ & $124(14,5)$ \\
P. Boizinho & & & & \\
$30 / 04 / 1997$ & $70(5,1)$ & $47(5,4)$ & $124(8,8)$ \\
$05 / 06 / 1997$ & $128(45,9)$ & $64(13,3)$ & $122(16,0)$ \\
$15 / 09 / 1997$ & $83(12,3)$ & $75(13,6)$ & $110(10,7)$ \\
B. da Ribeira & $33(3,6)$ & $36(1,4)$ & $36(1,8)$ \\
$*$ & & & & \\
\hline
\end{tabular}

(*) média das concentraçōes encontradas nas 3 coletas realizadas

Os resultados obtidos demonstraram que, através das concentrações nas espécies de macrófitas marinhas analisadas, pode-se caracterizar a disponibilidade de metais para a biota em ecossistemas costeiros sujeitos à contaminação por metais pesados, como a Baía de Sepetiba. As concentrações nas algas indicaram distribuição espacial ampla dos metais Zn e Cd na Baía de Sepetiba e evolução temporal (1990-1997) não sazonal e relacionada com a carga de lançamentos de metais para a Baía. Os resultados do experimento de transplante mostraram que Padina gymnospora acumulou os metais $\mathrm{Zn}$ e Cd em período curto de tempo (um mês), refletindo a biodisponibilidade dos metais no ambiente, complementando o monitoramento com as populações naturais. Em relação à monocotiledônea Halodule wrightii, evidenciou-se que as raízes e folhas são os principais compartimentos para acumulação de metais e que concentrações elevadas nas raízes podem refletir a biodisponibilidade de metais quando alterações no sedimento ocorrerem, como por exemplo, a dragagem para ampliação do Porto de Sepetiba.

\section{Agradecimentos}

Ao PRONEX/MCT “Impactos Antrópicos em Ambientes Aquáticos”, CNPq (no. 521688/96-5 Oc.) e FAPERJ (E-26/170.336/98), pelos auxílios concedidos para execução deste trabalho.

\section{Referências bibliográficas}

Amado Filho, G. M.; Andrade, L. R.; Karez, C.S.; Yoneshigue-Valentin, Y. \& Pfeiffer, W.C. 1997a . Effects on growth and accumulation of zinc in six seaweed species. Ecotoxicology and Environmental Safety 37: 223-228. 
Amado Filho, G. M.; Andrade, L. R.; Reis, R. P.; Bastos, W. \& Pfeiffer, W. C. 1997b. Heavy metal concentration in seaweed species from the Abrolhos Reef region, Brazil. Proceedings of the VIII International Coral Reef Symposium 2: 1843-1846.

Amado Filho, G. M.; Andrade, L. R.; Karez, C.S \& Pfeiffer, W. C. 1997c. Zinc and cadmium accumulation by Padina gymnospora (Phaeophyceae) from Sepetiba Bay (Rio de Janeiro, Brazil) over the last 8 years. VII Congresso Latino-Americano sobre Ciências do Mar 1: 28-30.

Barcellos, C.; Rezende, C. E. \& Pfeiffer, W. C. 1991. Zn and Cd production and pollution in a Brazilian coastal region. Marine Pollution Bulletin 22(11): 558-561.

Eide, I.; Adjers, K.; Ruokolahti, C. \& Bondestam, M. 1980. Long term uptake and release of heavy metals by Ascophyllum nodosum (L.) Les Jolis (Phaeophyceae) in situ. Environmental Pollution 23: 19-28

Karez, C. S.; Magalhães, V. F.; Pfeiffer, W.C. \& Amado Filho, G. M. 1994 a . Trace metal accumulation by algae in Sepetiba Bay, Brazil. Environmental Pollution 83: 351-356.

Karez, C. S.; Amado Filho, G. M.; Moll, D. M. \& Pfeiffer, W. C. 1994b . Concentrações de metais em algas marinhas bentônicas de três regiōes do Estado do Rio de Janeiro. Anais da Academia Brasileira de Ciências 66(2): 205-211.

Lacerda, L. D; Teixeira, V. L. \& Guimarāes, J. R. 1985. Seasonal variation of heavy metals in seaweeds from Conceição de Jacareí (RJ) Brazil. Botanica Marina 28: 339-343.

Lacerda, L. D. \& Rezende, C. E. 1986. Metals in the seagrass Halodule wrightii Aschers during one growing season. Revista Brasileira de Botânica 9: 87-90.

Lacerda, L. D.; Pfeiffer, W. C. \& Fizsman, M. 1987. Heavy metal distribution, availability and fate in Sepetiba Bay, S.E. Brazil. The Science of Total Environment 65: 163-173.

Multiservice. 1997. Projeto de dragagem do canal de acesso ao Porto de Sepetiba - RJ. Cia Docas do Rio de Janeiro. Relatório de Impacto Ambiental (RIMA), Rio de Janeiro.

Nicolaidou, A. \& Nott, J. A. 1998. Metals in sediment, seagrass and gastropods near a nickel smelter in Greece: possible interactions. Marine Pollution Bulletin 36(5): 360-365.

Pulich, W. M. 1980. Heavy metal accumulation by selected Halodule wrightii Aschers populations in the Corpus Christi Bay area. Contribution in Marine Science 23: 89-100.

Pulich, W. M. 1982. Edaphic factors related to Shoalgrass (Halodule wrightii Aschers) production. Botanica Marina 25: 467-475

Riget, F; Johansen, P. \& Asmund, G. 1995. Natural seasonal variation of cadmium, copper, lead and zinc in brown seaweed (Fucus vesiculosus). Marine Pollution Bulletin 30(6): 409-413.

Thursby, G. B. 1984. Root-exuded oxygen in the aquatic angiosperm Ruppia maritima. Marine Ecology Progress Series 16: 303-305. 